\title{
Process analysis of waste bamboo materials using solvent liquefaction
}

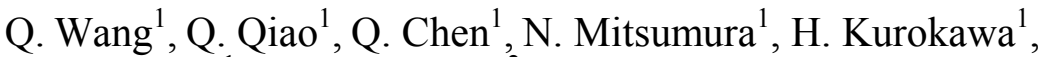 \\ K. Sekiguchi ${ }^{1} \&$ K. Sugiyama ${ }^{2}$ \\ ${ }^{I}$ Graduate School of Science and Engineering, Saitama University, Japan \\ ${ }^{2}$ Hachinohe National College of Technology, Japan
}

\begin{abstract}
Bamboo is one of the most significant biomass resources which has been used in houses, flooring, construction of scaffolding and bridges, etc. The solvent liquefaction process is one promising technique for effective utilization of waste bamboo materials for the lignocelluloses which can be converted to liquid reactive materials as biomass-based materials. Bamboo has the advantage of providing liquefied products with a small range of variances. The components of bamboo have high acidity in the presence of mineral acid catalysts and possess constituents which can react with polyethylene glycol 400 (PEG 400). In this study, waste bamboo materials have been used in liquefaction experiments. The liquefaction process and liquefied residue have been measured according to the liquefied conditions and the surface changes of waste bamboo samples observed by a scanning electron microscope. The changes in the functional groups have been analysed by a Fourier transform infrared spectrometer and the behavior of the crystalline structures of liquefied bamboo has been determined by X-ray diffraction. Other experiments, such as the degree of polymerization, have also been carried out to confirm the results. It was found that increments of the temperature and the amount of the acid catalysts improved the efficiency of liquefaction. Meanwhile, the dissolution time of lignin was significantly shorter than that of cellulose in the solvent liquefaction process of PEG 400.

Keywords: bamboo, liquefaction, biomass-based materials, FT-IR, X-ray diffraction, SEM.
\end{abstract}




\section{Introduction}

Bamboo, as a significant biomass resource, has been used in many fields of biomass utilization. It is also extensively used to make furniture, food steamers, chopsticks, paper pulp, etc. Many kinds of bamboo grow naturally in tropical, subtropical, and temperate regions around the world. Bamboo is fast-growing, economic and has a high cultural significance in East Asia and South-Eastern Asia. The solvent liquefaction process is one promising technique for effective utilization of bamboo for the lignocelluloses which can be converted to liquid reactive materials as biomass-based materials. Liquefaction technology, as one effective method of using the lignocellulosic biomass, has been considered for preparation of biomass-based materials. In early studies of organic solvent liquefaction, Lin et al. [1] also attempted to explain the reaction liquefaction mechanism of cellulose with phenol solvent under acid-catalyzed conditions. They found that the yield of various compounds was dependent on the reaction conditions. Therefore, the structure and properties of liquefied products in the later stage of liquefaction may be controlled by adjusting these reaction conditions or time. As the target product, biomass-based polyurethane films and Novolak phenol foam resins were prepared, and their structures and properties discussed [2, 3]. The research of bamboo liquefaction in this area is still relatively small. Moreover, it is known that bamboo fiber has high strength, low elongation, and high crystallinity. Moreover, the high crystallinity leads to the solvent liquefaction becoming slower and more inefficient. It takes longer to liquefy a biomass with higher crystalline index such as bamboo material [4]. Therefore, it is necessary to understand the change of waste bamboo materials in the liquefaction process so as to promote the liquefaction process and increase efficiency. Nevertheless, the mechanism of the formation of the condensed residues still needs to be clarified [5] and the research driving to overcome the problems with the drawbacks of the physical and mechanical properties of resin products from liquefied biomass [6].

In this study, waste bamboo materials were used in liquefaction experiments. The liquefaction process and liquefied residue were measured according to the conditions and the surface changes of the waste bamboo materials have been observed by a scanning electron microscope (SEM). The changes in the functional groups were analyzed by a Fourier transform infrared (FT-IR) spectrometer and influences on the crystalline structures of liquefied bamboo were determined by X-ray diffraction (XRD).

\section{Materials and methods}

\subsection{Materials}

Waste bamboo materials such as used bamboo chopsticks were collected from waste treatment and recycling factories of Japan. The experimental samples of cellulose (CAS No: 9004-34-6 MP Blomedicais, LLC, Co, Ltd., Japan) and lignin (Alkaline, CAS No: 9005-53-2 TCI. Co, Ltd., Japan) were used as the 
simulated bamboo material. The oven-dried waste bamboo powder was milled in smashing equipment and retained at a size range below $0.5 \mathrm{~mm}$, and then used as the samples of waste bamboo powder for the experiments. All the samples of waste bamboo powder were dried in an oven at $105^{\circ} \mathrm{C}$ for 24 hours. The main different acidic catalysts such as sulfuric acid (95 wt.\%), PEG 400 and other reagents were prepared with the analytical grade reagents made by Wako Pure Chemicals, Co. Ltd., Japan in accordance with the Japanese Industrial Standard (JIS).

\subsection{Chemical composition of waste bamboo materials}

In the proximate and ultimate analysis, the ash content of the residue left after the combustion of the bamboo samples was found according to the industrial standard method (JIS-M8812). $14.4 \mathrm{wt} \%$ of moisture content in the bamboo sample was determined by measuring the weight loss after drying the sample at $105^{\circ} \mathrm{C} .3 .8 \mathrm{wt} . \%$ of ash was measured by heating the bamboo powder to $900^{\circ} \mathrm{C}$ under carefully controlled conditions. Solubilities of $29.6 \mathrm{wt} \%$ in $1 \% \mathrm{NaOH}$ solution and 11.9 wt.\% in methanol-benzene were determined in this study. As the components of bamboo, 68.9 wt. $\%$ of holocellulose and 18.9 wt. $\%$ of lignin were present.

\subsection{Liquefaction experimental method}

The samples of waste bamboo powder were used in liquefaction experiments and the weight charge ratio of bamboo and PEG 400 was $1 / 4$, and the reaction temperatures were $120^{\circ} \mathrm{C}$ and $150^{\circ} \mathrm{C}$ by oil bath. The raw materials were placed in a $500 \mathrm{~mL}$ three necked flask equipped with a stirrer. Reactants were treated with methanol as the diluent. Different amounts of catalyst from 3 wt.\% to 10 wt.\% were added to the reactant as the reaction start time, and the methanol was added to the liquefied products as the reaction stop time.

\subsection{Measurement of liquefaction residue}

The liquefied bamboo samples were weighted and diluted with methanol, and Whatman filter papers (No. 40) were used to collect the liquefied residue. Here, the solvent-insoluble residue in equation (1) means the weight of liquefied residue through filtration which was diluted by methanol from the liquefied products, and the raw material means the weight of the reactants. The liquefied residue was dried by heating in an oven at $105^{\circ} \mathrm{C}$ for 12 hours and the residues calculated by equation (1).

$$
\text { Liquefied ratio }(\text { wt. } \%)=(\text { Solvent-insoluble residue } / \text { Raw material }) \times 100
$$

\subsection{Determination of the degree of polymerization}

About $0.04 \mathrm{~g}$ of liquefied bamboo under different experimental conditions were dissolved in $10 \mathrm{~mL}$ of cupriethylenediamine hydroxide solution. Then, the 
intrinsic viscosity of the solution was measured three times by a handmade Ubbelodhe viscometer, and the degree of polymerization (DP) was calculated according to the flow time. All the measurement experiments were carried out more than 3 times for each average value.

\subsection{Fourier transform infrared spectrometer analysis}

Several liquefied bamboo samples were analyzed by using a FT-IR spectrometer (FT-IR-6100 Jasco, Co. Ltd., Japan). The ratio of liquefied bamboo samples and spectroscopic grade $\mathrm{KBr}$ was 1:100; the time of mixing of the sample was more than one hour for full mixing. All of the infrared spectra were recorded in absorbance units in the $4000-400 \mathrm{~cm}^{-1}$ range.

\subsection{Scanning electron microscope analysis}

The surface changes of native and liquefied bamboo were observed by an Hitachi SEM (Model S-2400, Hitachi Co. Ltd., Japan) at an acceleration voltage of 15 $\mathrm{kV}$.

\subsection{X-ray diffraction analysis}

Crystalline structures of bamboo and liquefied bamboo were analyzed by an Ultima III X-Ray Diffractometer (Rigaku Co. Ltd., Japan). Ni-filtered $\mathrm{Cu} \mathrm{K} \alpha$ radition $(\lambda=0.1542 \mathrm{~nm})$ generated at a voltage of $40 \mathrm{kV}$ and a current of $40 \mathrm{~mA}$ was used. Intensities in the range from $10^{\circ}$ to $40^{\circ}$ with $4^{\circ} / \mathrm{min}$ scan speed were set for a total X-ray diffraction (XRD) analysis experiment. The crystallite height 200 (h200) and amorphous height (ham) was used to calculate the crystalline index (CI) by following equation (2).

$$
\text { Crystalline index }(\mathrm{CI})(\%)=\left(\frac{\mathrm{h}_{200}-\mathrm{h}_{\mathrm{am}}}{\mathrm{h}_{200}}\right) \times 100
$$

\section{Results and discussions}

\subsection{Residues results in different experimental conditions}

The amount of liquefied residue (LR) was a signification index to evaluate the performance of liquefied bamboo in solvent liquefaction. Two variables were used in the experiment. One was the different amount of sulfuric acid, the other was the different reaction temperature. These two response variables had an impact on the liquefaction results. Figure 1 shows the liquefaction rate of bamboo related to different amounts of sulfuric acid within $90 \mathrm{~min}$ at $120^{\circ} \mathrm{C}$. A greater quantity of sulfuric acid was added to the reactant. However, fewer residues were not obtained. On the contrary, the residues increased from $30 \mathrm{~min}$. The cellulose gradually converted to levulinic acid derivatives, following the conversion of lignin into solvent-soluble compounds in the early stages of bamboo liquefaction. Then, the mixture of levulinic acid derivatives and aromatic compounds reacted and converted to the solvent-soluble residue. The 
results indicated that the insoluble residue was formed when cellulose and lignin coexisted [7]. A condensation reaction had occurred during the liquefaction process. The experimental conditions were changed such as the solvent/cellulose addition ratio [8].

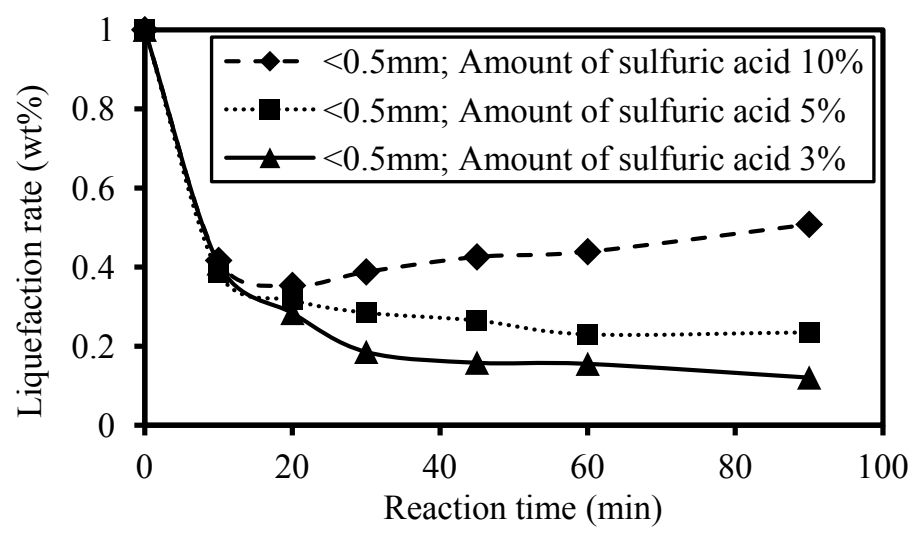

Figure 1: Liquefaction rate of waste bamboo materials with different amounts of sulfuric acid within $90 \mathrm{~min}$ at $120^{\circ} \mathrm{C}$.

When 5 wt.\% sulfuric acid of solvent was added into the experiment, more residues were obtained which also indicated that the insoluble residue was contained in the liquefied products. This problem will affect the mechanical properties of resin products from liquefied biomass [6].

Figure 2 shows the liquefaction rate of bamboo with different reaction temperatures within $90 \mathrm{~min}$ and $5 \mathrm{wt} . \%$ of catalysis. It was found that the fewer residues obtained at higher temperature and the yield of various compounds were dependent on the reaction conditions. Therefore, the structure and properties of liquefied products in the later stages of liquefaction may be controlled

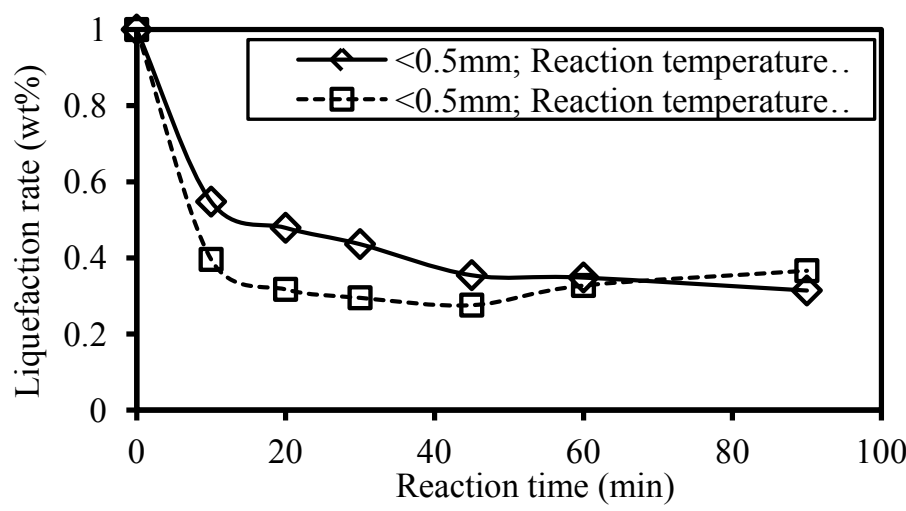

Figure 2: Liquefaction rate of waste bamboo materials with different reaction temperature within $90 \mathrm{~min}$ and $5 \mathrm{wt}$ \% of catalysis. 
by altering the reaction time and temperature [1]. However, the condensation reaction occurred in the high-temperature reaction. Therefore, bamboo liquefaction, application of lower temperatures and less amounts of additional acid were suggested to prevent the condensation reaction.

\subsection{FT-IR analysis}

In order to understand the change in the functional groups in bamboo, liquefied bamboo was observed by a FT-IR spectrometer. The major functional groups of liquefied products are listed in table 1. Compared with the peak of bamboo and its 5 min liquefied residues, a similar peak could be observed. The result indicates that there was still much lignin present in the untreated residues.

The FT-IR spectrometer of cellulose as a model substance is also shown in Figure 3. The peak was similar to the peak of bamboo $30 \mathrm{~min}$ residues. This result represented that less lignin was contained in the residues and proved that acid decomposition of lignin was faster than cellulose decomposition. For the detailed information, the absorption band from $3500 \mathrm{~cm}^{-1}$ to $3100 \mathrm{~cm}^{-1}$ was assigned as hydroxide, and the band of liquefied bamboo residues at $2908 \mathrm{~cm}^{-1}$ and $2907 \mathrm{~cm}^{-1}$ were assigned as the $\mathrm{CH}$ and $\mathrm{CH}_{2}$ stretching vibrations of aliphatic hydrocarbons. Two small peaks at $1743 \mathrm{~cm}^{-1}$ and $1736 \mathrm{~cm}^{-1}$ were assigned as $\mathrm{C}=\mathrm{O}$ bending which was attributable to the acetyl group or carboxyl group. The band at $1716 \mathrm{~cm}^{-1}$ was assigned as $\mathrm{C}=\mathrm{O}$ which was attributable to the carboxyl group stretching the conjugated carbonyl group. Two obvious peaks at $1600 \mathrm{~cm}^{-1}$ and $1510 \mathrm{~cm}^{-1}$ were assigned as $\mathrm{C}=\mathrm{C}$ bending which was attributable to the aromatic ring of the lignin. Another important band at $1151 \mathrm{~cm}^{-1}$ was assigned as $\mathrm{C}-\mathrm{O}-\mathrm{C}$ which was attributable to the $\mathrm{C}-\mathrm{O}-\mathrm{C}$ symmetric stretching vibration of the lignin $\beta-\mathrm{O}-4$ ether bond.

Table 1: Functional groups of waste bamboo materials and liquefied bamboo.

\begin{tabular}{|c|c|c|c|}
\hline $\begin{array}{l}\text { Absorption } \\
\text { Band }\left(\mathrm{cm}^{-1}\right)\end{array}$ & $\begin{array}{l}\text { Assign- } \\
\text { ment }\end{array}$ & Origin From & Reference \\
\hline $3500-3100$ & $\mathrm{O}-\mathrm{H}$ & Hydroxide & \\
\hline $2908 ; 2907$ & $\mathrm{CH} ; \mathrm{CH}_{2}$ & $\begin{array}{l}\mathrm{CH} \text { and } \mathrm{CH}_{2} \text { stretching vibration of } \\
\text { aliphatic hydrocarbons }\end{array}$ & [9] \\
\hline $1743 ; 1736$ & $\mathrm{C}=\mathrm{O}$ & Acetyl group or carboxyl group & \\
\hline 1716 & $\mathrm{C}=\mathrm{O}$ & $\begin{array}{l}\text { Carboxyl group stretching } \\
\text { conjugated carbonyl group }\end{array}$ & {$[10]$} \\
\hline $1600 ; 1510$ & $\mathrm{C}=\mathrm{C}$ & The aromatic ring of the lignin & [11] \\
\hline $\begin{array}{l}1605 ; 1508 ; \\
1423\end{array}$ & $\mathrm{C}-\mathrm{H}$ & $\mathrm{C}-\mathrm{H}$ of the aromatic ring & {$[10]$} \\
\hline $1430 ; 1370$ & $\mathrm{C}-\mathrm{H}$ & It is a group of $\mathrm{CH}_{2}$ & [12] \\
\hline 1151 & $\mathrm{C}-\mathrm{O}-\mathrm{C}$ & $\begin{array}{l}\text { COC symmetric stretching } \\
\text { vibration of lignin } \beta-O-4 \text { ether bond }\end{array}$ & [9] \\
\hline 1100 & $\mathrm{C}-\mathrm{O}$ & C-O functional group of cellulose & {$[12]$} \\
\hline 830 & $=\mathrm{C}-\mathrm{H}$ & Aromatic C-H & \\
\hline
\end{tabular}




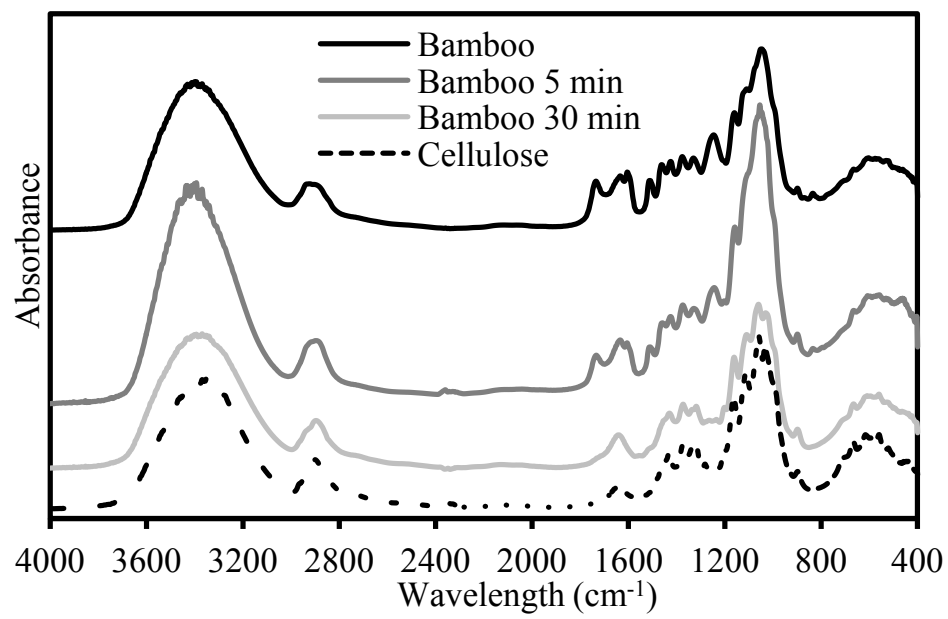

Figure 3: $\quad$ FT-IR spectrometer analysis of liquefied bamboo within $30 \mathrm{~min}$ at $120^{\circ} \mathrm{C}$.

\subsection{Surface morphological observation}

Figure 4 shows the SEM image of liquefied bamboo residues (LRs) related to reaction time within $30 \mathrm{~min}$ at $120^{\circ} \mathrm{C}$.
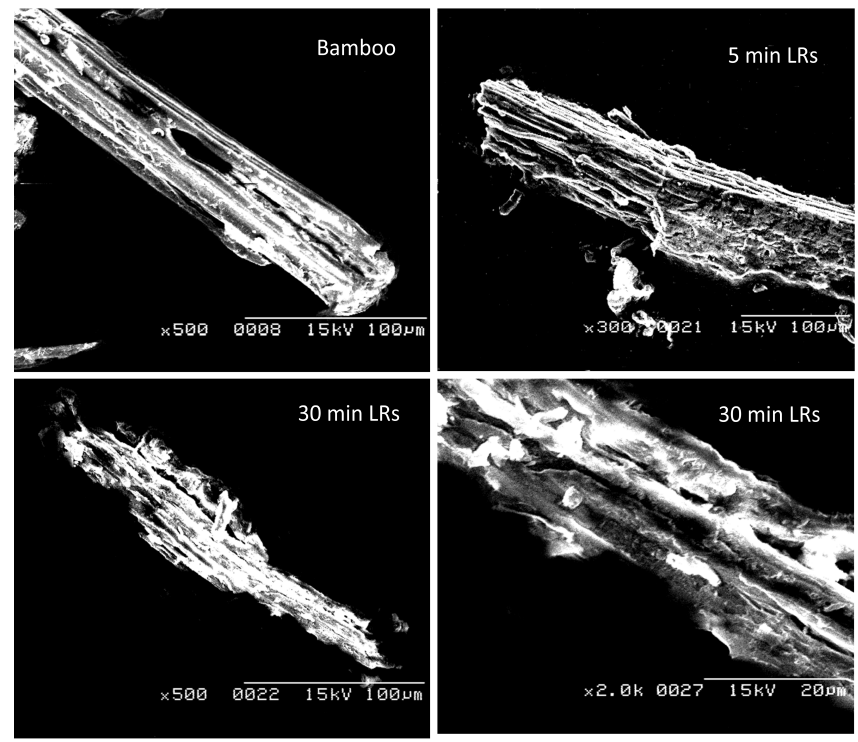

Figure 4: $\quad$ SEM photos of liquefied bamboo residues (LRs) related to reaction time within $30 \mathrm{~min}$ at $120^{\circ} \mathrm{C}$. 
The samples were the raw waste bamboo material, 5 min LRs and $30 \mathrm{~min}$ LRs. The different samples which had been obtained according to the reaction time were compared in the liquefaction process. Partial destruction was observed in 5 min LRs. The liquefied cellulose particles were not dissolved uniformly from the SEM image. This randomness made the speed of liquefied bamboo irregular. Most of the waste bamboo powder samples had been liquefied. Due to the condensation reaction, the sample was not observed after $30 \mathrm{~min}$.

\subsection{X-ray diffraction analysis}

X-ray diffraction gives the direct results and useful information to explain the structural changes of waste bamboo materials and liquefied bamboo. Generally, macro- and micro-structures, especially the degree of crystallinity, were changed drastically by changing the conditions of liquefaction. In this study, obvious structural changes were found (Figure 5). The apparent crystallinity increased at the beginning of liquefaction, but after 5 min the apparent crystallinity decreased with the liquefaction time. Such results are not in line with a general understanding. Therefore, a model substance was used to explain this phenomenon. The cellulose, lignin and the mixture of cellulose:lignin (2:1) was measured by XRD. It was found that the mixture of cellulose and lignin (2:1) had the lower apparent crystallinity (Figure 6).

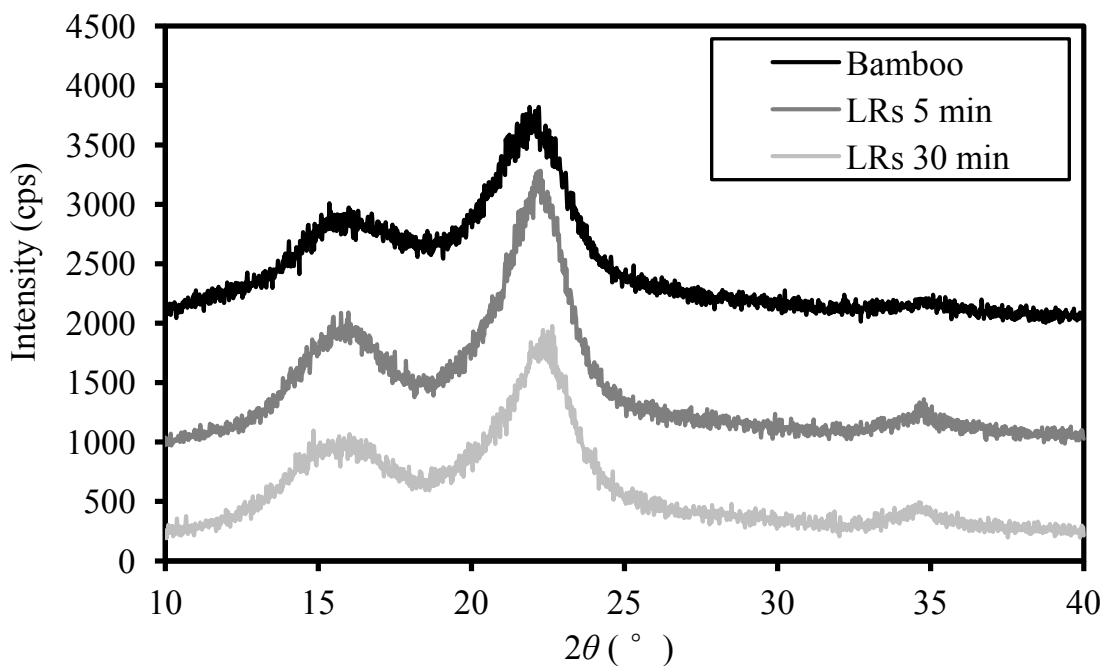

Figure 5: X-ray diffractograms of liquefied bamboo residues related to reaction time within $30 \mathrm{~min}$ at $120^{\circ} \mathrm{C}$. 


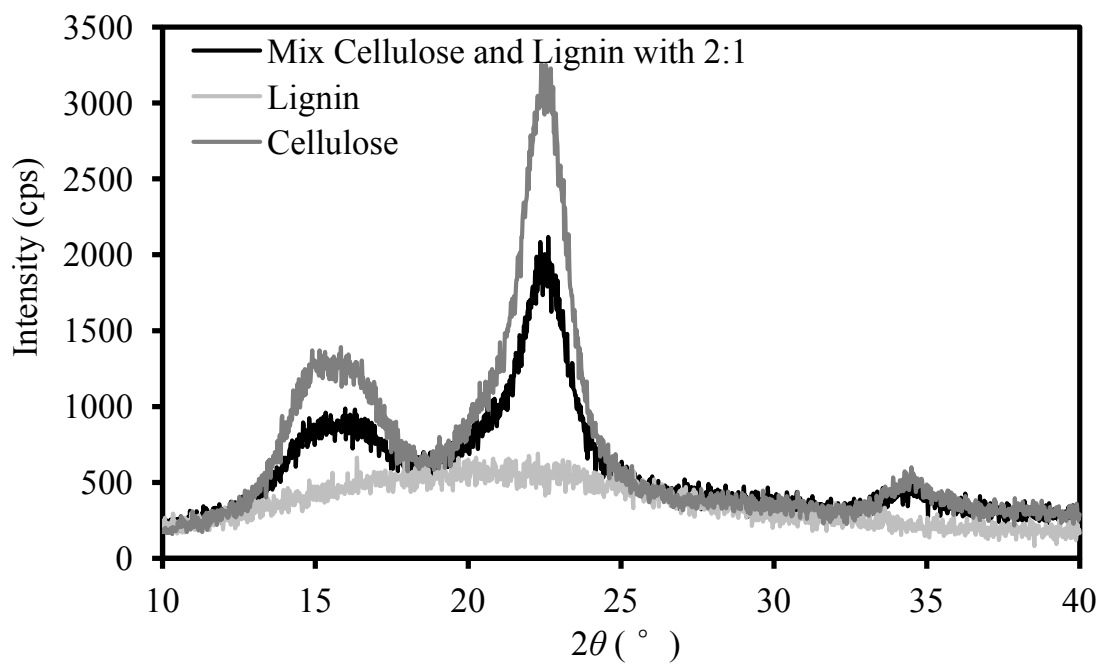

Figure 6: X-ray diffractograms of cellulose and lignin model substance.

This result did not mean that the apparent crystallinity of cellulose decreased because no reaction had taken place during mixture. One reason which can explain the phenomenon is that lignin dispersed the $\mathrm{x}$-ray diffraction. The misunderstood result showed the false appearance that the apparent crystallinity had decreased. Therefore, waste bamboo material contains $18.9 \%$ lignin before liquefaction. After liquefaction, the acid decomposition of lignin was faster than cellulose decomposition [13]. In other words, the peak of 5 min LRs was large because the content of cellulose was increased. Then, the intensity peak of 30 min became weaker. This result indicated that cellulose had been liquefied and the crystalline structure had been broken. Meanwhile, the long chain became short; the degree of polymerization also became low.

The change of apparent crystallinity related to different reaction temperatures within $30 \mathrm{~min}$ is illustrated in Figure 7. More apparent crystallinity occurred for higher temperatures of the liquefaction process. This result suggested that temperature was a significant factor to promote the decomposition of lignin. However, it did not play a significant role in the decomposition of cellulose. If we compare this result with the different amounts of acid catalyst, the greater amounts of acid catalyst promote the decomposition of cellulose and also contribute to the acid decomposition reaction [14].

\section{Conclusion}

Waste bamboo materials were used in this liquefaction experiment. The liquefaction process and liquefied residual were measured according to the conditions and morphological surface observed by a SEM. The change in the functional groups was analyzed by a FT-IR spectrometer and the effects on the crystalline structures of liquefied bamboo were determined by XRD. The 


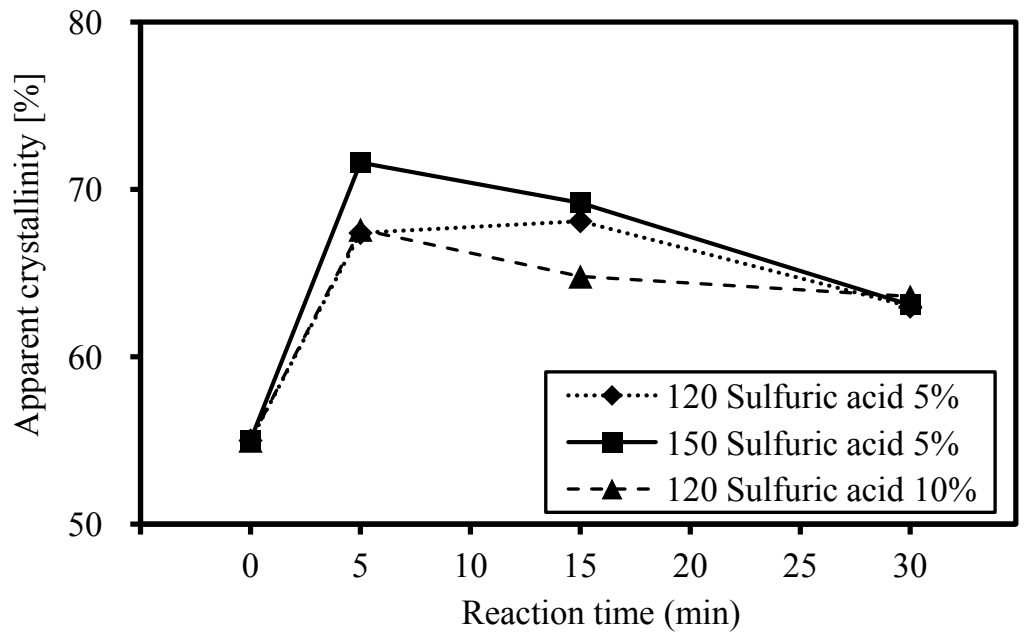

Figure 7: The changes of apparent crystallinity related to different reaction temperatures within $30 \mathrm{~min}$.

condensation reaction had occurred in the high-temperature reaction. Lower temperature and additions of lower amounts of acid were suggested to prevent the condensation reaction of bamboo liquefaction. The SEM image of the bamboo liquefaction process was presented for the first time. The liquefied cellulose particles did not dissolve uniformly as seen from the image. This randomness made the speed of liquefaction of bamboo irregular. According to the results of $\mathrm{XRD}$, it could be concluded that temperature was a significant factor to promote the decomposition of lignin and the higher amount of acid catalyst promoted the decomposition of cellulose. The results of FT-IR spectroscopy proved that acid decomposition of lignin was faster than cellulose decomposition. All the above results suggest that waste bamboo materials can be used for liquefaction but it is necessary to consider the energy efficiency as its high crystalline index leads to a long liquefaction time.

\section{Acknowledgements}

Some works of this study were supported by the special funds for Basic Research (B) (No. 22404022, FY2010 2012) of a Grant-in-Aid for Scientific Research of the Japanese Ministry of Education, Culture, Sports, Science and Technology (MEXT), Japan.

\section{References}

[1] Lin, L.Z., Yao, Y.G., Yoshida, M., Shiraishi, N., 2004. Liquefaction mechanism of cellulose in the presence of phenol under acid catalysis. Carbohydr. Poly., 57, 123-129. 
[2] Kurimoto, Y., Takeda, M., Doi, S., Tamura, Y., Ono, H., 2001. Network structures and thermal properties of polyurethane films prepared from liquefied wood. Bioresour. Technol. 77, 33-40.

[3] Lee, W.J., Chen, Y.C., 2008. Novolak PF resins prepared from phenol liquefied Cryptomeria japonica and used in manufacturing moldings. Bioresour. Technol., 99, 7247-7254.

[4] Yip, J., Chen, M., Szeto, Y.S., Yan, S., 2009. Comparative study of liquefaction process and liquefied products from bamboo using different organic solvents. Bioresour. Technol. 100, 6674-6678.

[5] Niu, min., Zhao, G.J., Alma, M.H., 2011. Polycondensation reaction and its mechanism during lignocellulosic liquefaction by an acid catalyst. For. Stud. China, 13, 71-79.

[6] Pan, Hui., 2011. Synthesis of polymers from organic solvent liquefied biomass: A review. Renew. Sust. Energ. Rev. 15, 3454- 3463.

[7] Kobayashi, M., Asano, T., Kajiyama, M., Tomita, B., 2005. Effect of ozone treatment of wood on its liquefaction. J. Wood. Sci. 51, 348-356.

[8] Zhang, Y.C., Ikeda, A., Hori, N., Takemara, A., Ono, H., Yamada, T., 2006. Characterization of liquefied product from cellulose with phenol in the presence of sulfuric acid. Bioresour. Technol. 97, 313-321.

[9] Wen-Jau Lee, Meng-Shinan Lin, Preparation and Application of Polyurethane Adhesives Made from Polyhydric Alcohol Liquefied Taiwan Acacia and China Fir, Journal of Applied Polymer Science, 2008, Vol. 109, 23-31.

[10] Kenji Izumo, Masami Fukushima, Influence of Wood Species on the Properties of Biopolyurethane Prepared from Liquefied Wood with Residue, Journal of Applied Polymer Science, 2010, Vol. 118, 2109-2115.

[11] Fangeng Chen, Zhuomin Lu, Liquefaction of wheat straw and preparation of rigid polyurethane foam from the liquefaction products, Journal of Applied Polymer Science, 2009, Vol. 111, 508-516.

[12] El-barbary M. Hassan, Nadia Shukry, Polyhydric alcohol liquefaction of some lignocellulosic agricultural residues, industrial crops and products, 27, 2008, 33-38.

[13] Kobayashi, M., Asano, T., Kajiyama, M., Tomita, B., 2004. Analysis on residue formation during wood liquefaction with polyhydric alcohol. $J$. Wood. Sci. 50, 407-414.

[14] Sung, P.M., Jeong, P.J., 2009. Liquefaction of cellulose in the presence of phenol using p-toluene sulfonic acid as a catalyst. J. Ind. Eng. Chem. 15, 743-747. 